\title{
Effect of high WDR5 expression on the hepatocellular carcinoma prognosis
}

\author{
ZHOUJUN CUI, HONGBO LI, FENG LIANG, CUILING MU, YUHUA MU, XUEGONG ZHANG and JUNDONG LIU
}

Department of General Surgery, People's Hospital of Rizhao, Rizhao, Shandong 276800, P.R. China

Received March 17, 2017; Accepted February 22, 2018

DOI: $10.3892 / \mathrm{ol} .2018 .8298$

\begin{abstract}
WD repeat domain 5 (WDR5) serves an important role in various biological functions through the epigenetic regulation of gene transcription. Aberrant expression of WDR5 has been observed in various types of human cancer, including prostate cancer, breast cancer and leukemia. However, the role of WDR5 expression and its clinical implications in hepatocellular carcinoma (HCC) remain largely unknown. The present study investigated the WDR5 expression pattern in HCC. It was demonstrated that the mRNA and protein levels of WDR5 were upregulated in HCC cancer tissues compared with normal adjacent tissues using reverse transcription-quantitative polymerase chain reaction and western blotting. Furthermore, the elevated WDR5 protein level was significantly associated with the histological grade $(\mathrm{P}=0.038)$, tumor size $(\mathrm{P}=0.023)$, tumor-node-metastasis stage $(\mathrm{P}=0.035)$ and reduced long-term survival time. Additionally, it was demonstrated through the shRNA-mediated knockdown of WDR5 in HCC cells in vitro that WDR5 expression promotes cell proliferation using an MTT assay. Taken together, the results suggested that WDR5 overexpression may have an oncogenic effect in HCC, and may be a promising biomarker for the diagnosis and prognosis of HCC.
\end{abstract}

\section{Introduction}

Hepatocellular carcinoma (HCC) is one of the most common types of cancer, causing $\sim 662,000$ cancer-associated mortalities worldwide per year (1). According to an epidemiological survey, HCC has a particularly high incidence in males, and is most common between the ages of 30 and 50 years $(2,3)$. In the majority of cases, the patients were either infected by viral hepatitis (type B or C) or exposed to metabolic toxins such as alcohol or aflatoxin (4). At present, the treatment

Correspondence to: Dr Hongbo Li, Department of General Surgery, People's Hospital of Rizhao, 126 Tai'an Road, Rizhao, Shandong 276800, P.R. China

E-mail: hbong_li@foxmail.com

Key words: hepatocellular carcinoma, WD repeat domain 5, histone H3 lysine 4, short hairpin RNA, prognosis options for patients with HCC include surgical resection, liver transplantation, chemoembolization and molecularly targeted therapy (5). However, the prognosis for patients with HCC remains poor (5). Therefore, it is important to investigate and explore the potential prognostic factors for HCC.

Post-translational modifications of histones, particularly the methylation of histones $\mathrm{H} 3$ and $\mathrm{H} 4$, regulate chromatin structure and function by promoting the assembly of protein complexes at specific genomic loci (6). The methylation of histone $\mathrm{H} 3$ lysine 4 (H3K4) is a highly conserved modification in the epigenetic regulation of eukaryotic transcription (6). The aberrant methylation of H3K4 by H3K4 methyltransferases (H3K4MTs) has been demonstrated to be associated with different types of cancer and developmental disorders $(7,8)$. In humans, the MLL/SET1 complexes, which are primarily responsible for $\mathrm{H} 3 \mathrm{~K} 4$ methylation, are composed of mixed lineage leukemia (MLL) proteins 1-4 and the SET domain containing 1A/B catalytic subunit. Additionally, the full enzyme activity of $\mathrm{H} 3 \mathrm{~K} 4 \mathrm{MTs}$ requires the interaction of the catalytic subunit with the core regulatory subunits, including WD repeat domain 5 (WDR5), retinoblastoma-binding protein-5 (RbBP5), absent small homeotic-2-like (ASH2L) and dumpy-30 (DPY-30) $(9,10)$. Although the precise structure of the assembled catalytic subunit and core regulatory subunits remains unclear, WDR5 is considered to be the key for the association of RbBP5, ASH2L and DPY-30 with MLL1, since RbBP5 and ASH2L do not stably interact with MLL1 in the absence of WDR5 (11). WDR5 is a highly conserved protein with a molecular weight of $\sim 36 \mathrm{kDa}$ that is composed of a short, unstructured N-terminus followed by seven WD40 repeats, which can adopt a seven-bladed $\beta$-propeller fold (12). Previous studies revealed that WDR5 can interact with HDAC3 to activate mesenchymal gene expression, and may promote epithelial-mesenchymal transition by H3K4 methylation $(11,12)$. Furthermore, it has been demonstrated that WDR5 is overexpressed in prostate cancer (13), bladder cancer (14), leukemia (15) and breast cancer (16). It has also been demonstrated that the overexpression of WDR5 may promote cell proliferation (13-16). Despite the increased understanding of WDR5, its expression pattern and association with phenotypic parameters in HCC remains unclear.

An important mechanism of HCC development is the accumulation of genetic and epigenetic alterations. Therefore, the aims of the present study were to measure the expression of WDR5 in HCC tumor and adjacent tissues, and investigate 
the association between its expression and clinicopathological prognostic variables. Furthermore, the association of WDR5 expression with the overall survival time of patients with $\mathrm{HCC}$ was evaluated.

\section{Materials and methods}

Patients and specimens. A total of 113 patients diagnosed with HCC and treated at the People's Hospital of Rizhao (Rizhao, China) between August 2004 and September 2011 were enrolled in the present study. The study was approved and monitored by the Research Ethics Committee of the People's Hospital of Rizhao. Informed written consent was obtained from all the participating patients, whose specimens were anonymized according to the ethical and legal standards of the Research Ethics Committee. All patients had no history of chemotherapy or radiotherapy prior to surgery. HCC tumors were surgically removed from the patients, snap-frozen in liquid nitrogen and stored at $-80^{\circ} \mathrm{C}$ until further usage. Adjacent noncancerous tissues were obtained from the distal edge of the resection, $\geq 2 \mathrm{~cm}$ from the tumor, and were also immediately frozen in liquid nitrogen and stored at $-80^{\circ} \mathrm{C}$. The tumor stage of each patient was reviewed and classified according to the 6th edition of the tumor-node-metastasis (TNM) classification of International Union Against Cancer (17). Histological grade was evaluated independently by pathologists according to the World Health Organization classification system (18). All patients completed follow-up for 5 years after surgery. The survival period was calculated using the date of the curative resection of the tumor as the first day, and the date of mortality as the final day. The clinical and pathological variables of the patients were collected and are presented in Table I.

Immunohistochemical (IHC) analysis. The paraffin-embedded HCC tumor and normal liver tissues were cut into 5- $\mu \mathrm{m}$ sections. These sections were deparaffinized in $100 \%$ xylene (Beyotime Institute of Biotechnology, Shanghai, China) and rehydrated using a series of graded alcohols (Sinopharm Chemical Reagent Co. Ltd., Shanghai, China). Antigen retrieval was performed by boiling the sections in $\mathrm{pH} 6.0$ citrate buffer (Beyotime Institute of Biotechnology) for $5 \mathrm{~min}$. Subsequently, these sections were blocked with $10 \%$ goat serum (OriGene Technologies, Inc, Beijing, China) for $1 \mathrm{~h}$ prior to incubation with the previously described primary antibody against WDR 5 at $4^{\circ} \mathrm{C}$ for $2 \mathrm{~h}$, followed by a horseradish peroxidase-conjugated goat anti-rabbit IgG (cat. no., PI-1000; dilution, 1:200; Vector Laboratories, Burlingame, CA, USA) at room temperature for $2 \mathrm{~h}$. The reaction was visualized using diaminobenzidine substrate chromogen solution (Gene Technology Biotechnology Co., Ltd., Shanghai, China). These sections were then counterstained with hematoxylin, and examined under an optical microscope. The scoring method was performed as previously described (19).

Cell culture. The SNU-449 and MHCC97H human HCC cell lines were purchased from American Type Culture Collection (Manassas, VA, USA) and cultured in RPMI-1640 medium (Thermo Fisher Scientific, Inc., Waltham, MA, USA) supplemented with $10 \%$ fetal bovine serum (FBS; Invitrogen; Thermo Fisher Scientific, Inc.). The HL-7702 normal liver cell line obtained from Cell Bank of Chinese Academy of Sciences (Shanghai, China) was cultured in Dulbecco's modified Eagle's medium (Thermo Fisher Scientific, Inc.) supplemented with $10 \%$ FBS. All cell lines were cultured at $37^{\circ} \mathrm{C}$ in a $5 \% \mathrm{CO}_{2}$ atmosphere.

Stable WDR5 knockdown in HCC cells. The short hairpin RNA (shRNA) targeting WDR5 and a non-targeting shRNA were designed and synthesized according to the study by Dai et al (16). The shRNA sequences were as follows: shRNA against WDR5, 5'-GCTCAGAGGATAACCTTGTTT-3'; non-targeting shRNA, 5'-CCTAAGGTTAAGTCGCCC TCG-3'. These shRNAs were cloned into the pSuper.retro. puro vector (Oligoengine, Seattle, WA, USA) and the generation of retrovirus and infection were conducted according to the manufacture's protocol. Stably transfected cell lines were established by selection with G418 $(600 \mu \mathrm{g} / \mathrm{ml})$. The knockdown of WDR5 expression in HCC cell lines was confirmed by reverse transcription-quantitative polymerase chain reaction (RT-qPCR) and western blot analysis. The cells were harvested for cell proliferation and migration assays.

Cell proliferation assay. The cell proliferation rate was analyzed using an MTT assay. The wild-type and transfected cell lines were seeded in 96-well plates at a density of $\sim 3 \times 10^{3}$ cells/well. MTT solution (20 $\mu \mathrm{l}$ of $5 \mathrm{mg} / \mathrm{ml}$ MTT, Invitrogen; Thermo Fisher Scientific, Inc.) was added to each well, and the plates were incubated for $4 \mathrm{~h}$ at $37^{\circ} \mathrm{C}$. Subsequently, $150 \mu \mathrm{l}$ dimethyl sulfoxide was added to each well and incubated for $10 \mathrm{~min}$ at $37^{\circ} \mathrm{C}$ to dissolve the formazan crystals. The optical absorbance was measured at $490 \mathrm{~nm}$ using a microplate reader (Thermo Fisher Scientific, Inc,) at the time points of 12, 24, 48 and $72 \mathrm{~h}$. Assays were repeated $>3$ times.

$R T$ - $q P C R$. Total RNA was prepared from the 113 tumor tissues, 113 adjacent non-cancerous tissues and the cell lines using an RNAiso Plus kit (Takara Bio, Inc., Otsu, Japan) according to the manufacturer's protocol. The cDNA synthesis was conducted using the PrimerScript RT Master mix (Takara Bio, Inc.) using the ABI ProFlex PCR system (Applied Biosystems; Thermo Fisher Scientific, Inc.). RT-qPCR was performed with qSTAR SYBR Master mix (OriGene Technologies, Inc., Rockville, MD, USA) and a StepOne Plus Real-Time PCR system (Applied Biosystems; Thermo Fisher Scientific, Inc.). Each experiment was performed in triplicate. The relative expression of WDR5 was calculated using the $2^{-\Delta \Delta C q}$ method (20). The thermocycling conditions were as follows: Initial denaturation at $95^{\circ} \mathrm{C}$ for $10 \mathrm{~min}$, followed by 40 cycles of denaturation at $95^{\circ} \mathrm{C}$ for $1 \mathrm{~min}$ and annealing/extension at $56^{\circ} \mathrm{C}$ for $1 \mathrm{~min}$. The transcription level of GAPDH was used as an internal control. The primers were designed using Primer 3.0 software and are listed as follows: WDR5, forward, 5'-AAT ATCCGATGTAGCCTGGTC-3', and reverse, 5'-TTGGAC TGGGGATTGAAGTTG-3'; GAPDH forward, 5'-CAAGGC TGAGAACGGGAAG-3', and reverse, 5'-TGAAGACGCCAG TGGACTC-3'.

Western blot analysis. The total protein was extracted from the collected cell lines, and the tumor and corresponding noncancerous tissue samples using the Whole Protein Extraction kit 
Table I. Associations between WDR5 tumor expression and clinicopathological parameters.

\begin{tabular}{|c|c|c|c|c|}
\hline \multirow[b]{2}{*}{ Variable } & \multirow[b]{2}{*}{ Cases, n } & \multicolumn{2}{|c|}{$\begin{array}{c}\text { WDR5 } \\
\text { expression }\end{array}$} & \multirow[b]{2}{*}{ P-value } \\
\hline & & High & Low & \\
\hline Sex & & & & NS \\
\hline Male & 62 & 40 & 22 & \\
\hline Female & 51 & 39 & 12 & \\
\hline Age (years) & & & & NS \\
\hline$\geq 50$ & 65 & 44 & 21 & \\
\hline$<50$ & 48 & 35 & 13 & \\
\hline $\operatorname{AFP}(\mathrm{ng} / \mathrm{ml})$ & & & & NS \\
\hline$>400$ & 52 & 38 & 14 & \\
\hline$\leq 400$ & 61 & 41 & 20 & \\
\hline HBsAg & & & & NS \\
\hline Negative & 54 & 37 & 17 & \\
\hline Positive & 59 & 42 & 17 & \\
\hline Liver cirrhosis & & & & NS \\
\hline No & 45 & 34 & 11 & \\
\hline Yes & 68 & 45 & 23 & \\
\hline Histological grade & & & & 0.038 \\
\hline Well/moderate & 53 & 32 & 21 & \\
\hline Poor & 60 & 47 & 13 & \\
\hline Tumor size & & & & 0.023 \\
\hline$\geq 5$ & 58 & 35 & 23 & \\
\hline$<5$ & 55 & 44 & 11 & \\
\hline TNM stage & & & & 0.035 \\
\hline I-II & 56 & 34 & 22 & \\
\hline III-IV & 57 & 45 & 12 & \\
\hline
\end{tabular}

WDR5, WD repeat domain 5; NS, not significant; AFP, $\alpha$-fetoprotein; HBsAg, hepatitis B surface antigen; TNM, tumor-node-metastasis.

(Fermentas; Thermo Fisher Scientific, Inc., Pittsburgh, PA, USA) following the manufacturer's protocol. The protein concentration was quantified using the Bradford method. Protein samples $(20 \mu \mathrm{g})$ were mixed with $6 \mathrm{X}$ SDS sample buffer containing $10 \% \beta$-mercaptoethanol and separated by $10 \%$ SDS-PAGE and then transferred onto polyvinylidene fluoride membranes (GE Healthcare Life Sciences, Chicago, IL, USA). The membranes were incubated in 5\% fat-free milk for $1 \mathrm{~h}$ at room temperature prior to the addition of antibodies. Primary antibodies against WDR5 (cat. no., ab178410; dilution, 1:1,000; Abcam, Cambridge, MA, USA) and GAPDH (cat. no., 2118; dilution, 1:1,000; Cell Signaling Technology, Inc., Danvers, MA, USA) were incubated with the membrane for $1 \mathrm{~h}$ at room temperature. Subsequent to washing with PBS with $0.5 \%$ Tween, the membranes were incubated with a horseradish peroxidase-conjugated goat anti-rabbit antibody (cat. no., 7074; dilution, 1:1,000; Cell Signaling Technology, Inc.) at room temperature for $1 \mathrm{~h}$. Bands were visualized with an Luminata ${ }^{\mathrm{TM}}$ Western HRP Substrates (EMD Millipore, Billerica, MA, USA). Densitometry was performed using
Image J software (version 1.37; National Institutes of Health, Bethesda, MA, USA). GAPDH protein level was used as a loading control.

Statistical analysis. SPSS version 20.0 software (IBM Corporation, Armonk, NY, USA) was used to conduct all statistical analyses. The $\chi^{2}$ test was used to analyze the association between WDR5 expression and clinicopathological variables. The survival rate was calculated using the Kaplan-Meier method, and analyzed with the log-rank test. The univariate and multivariate Cox proportional hazards regression model analyses were conducted to identify the factors that were significant for the prognosis of HCC patients. Student's t test was used to analyze the statistical difference between two groups. One-way analysis of variance with a Tukey post-hoc test was used to analyze the statistical difference among multiple groups. Results are expressed as the mean \pm standard error of the mean. $\mathrm{P}<0.05$ was considered to indicate a statistically significant difference.

\section{Results}

WDR5 is overexpressed in HCC. To investigate whether WDR5 is associated with the development and progression of HCC, WDR5 mRNA and protein expression levels were measured in 113 HCC tissues and 113 matched normal adjacent tissues by RT-qPCR and western blot analyses. Compared with the normal adjacent liver tissues, the HCC tissues exhibited a significant increase in WDR5 expression $(\mathrm{P}<0.05$; Fig. 1A and B). The majority of the HCC cancer tissues $(69.9 \%, 79 / 113$ cases) exhibited high WDR5 expression, whereas the majority of the normal tissues $(75.2 \%$, 85/113 cases) exhibited low WDR5 expression (Fig. 1C). These results demonstrated that WDR5 was upregulated in HCC tissues.

Furthermore, the WDR5 expression in HCC tissues and matched normal adjacent tissues was measured by IHC. The level of WDR5 expression in HCC tissues was markedly higher than in the normal adjacent liver tissues (Fig. 1D and E). Based on the WDR5 expression analysis results obtained from RT-qPCR, western blot analysis and IHC, the patients were divided into two groups: Low- and high-WDR5 expression groups (cut-off value: 1.39).

Association between WDR5 expression and clinicopathological variables. The association between WDR5 expression and the clinicopathological features of 113 patients with HCC was studied (Table I). High WDR5 expression in HCC was significantly associated with histological grade $(\mathrm{P}=0.038)$, tumor size $(\mathrm{P}=0.023)$ and $\mathrm{TNM}$ stage $(\mathrm{P}=0.035)$, but not with sex, age, $\alpha$-fetoprotein (AFP) level, hepatitis B surface antigen (HBsAg) or liver cirrhosis (all $\mathrm{P}>0.05$ ). These findings suggested that WDR5 overexpression may contribute to HCC progression.

WDR 5 overexpression promotes cell proliferation. In order to examine the role of WDR5 in HCC, the expression of WDR5 in HCC cell lines and normal liver cell lines was examined. As presented in Fig. 2A and B, the mRNA and protein expression levels of WDR5 were markedly higher in HCC cells 

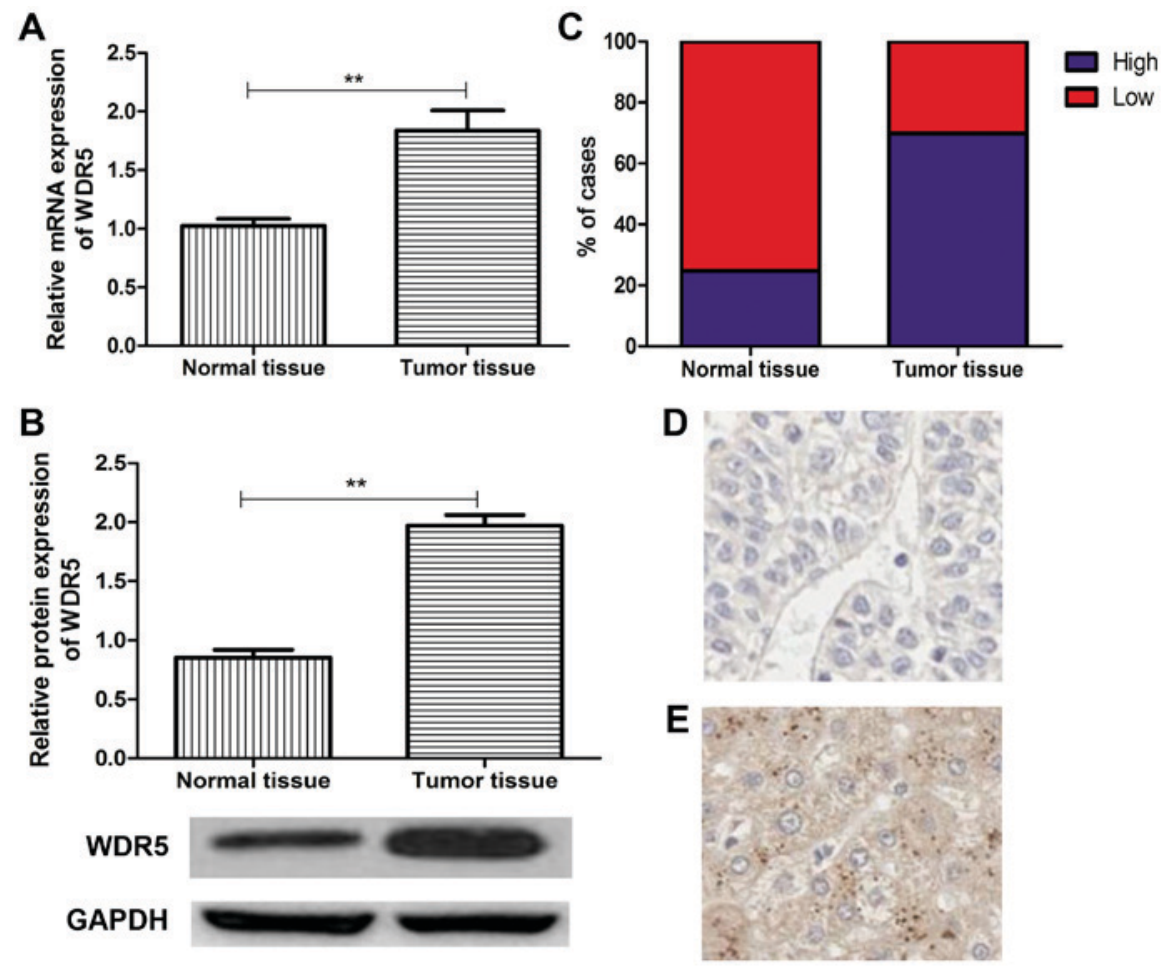

Figure 1. Significantly elevated WDR5 expression levels in HCC tumor tissue compared with adjacent non-tumor tissue. (A) Reverse transcription-quantitative polymerase chain reaction and (B) western blotting were used to analyze WDR5 expression in the tumor and adjacent samples. (C) Percentages of HCC tissues and adjacent non-tumor tissues high and low WDR5 expression. Immunohistochemistry was used to analyze the WDR5 expression in (D) adjacent and (E) tumor tissue (magnification, x200). Statistical analysis was performed with Student's t-test. ${ }^{* *} \mathrm{P}<0.01$. WDR5, WD repeat domain 5; HCC, hepatocellular carcinoma.

than in normal liver cells. WDR5-knockdown HCC cell lines were created with WDR5-shRNA. To verify the efficacy of WDR5-specific shRNA, a non-targeting shRNA was transfected into the HCC cell lines, and the mRNA expression level was evaluated with RT-qPCR. As displayed in Fig. 2C, it was demonstrated that the WDR5-shRNA effectively downregulated the expression of WDR5 in the HCC cell lines in vitro, whereas the non-targeting shRNA had no significant effect on WDR5 compared with untransfected cells.

Furthermore, the effect of WDR5 overexpression on HCC cell lines was analyzed with an MTT assay. As shown in Fig. 3A, the proliferation rates of HCC cell lines were evidently higher than the normal liver cell line. The HCC cells transfected with non-targeting shRNA and untransfected HCC cells had similar proliferation rates, as shown in Fig. 3B-D. Conversely, the HCC cell lines transfected with WDR5-specific shRNA exhibited a significantly lower proliferation rate than untransfected HCC cells or HCC cell lines transfected with non-targeting shRNA. Taken together, these results suggest that the expression of WDR5 was enhanced in HCC cell lines, and that WDR5 expression may promote the proliferation of HCC cells in vitro.

Prognostic value of high WDR5 expression in HCC. Overall survival rates were analyzed to evaluate the prognostic role of WDR5 expression using Kaplan-Meier survival curves. The results demonstrated that the 5-year overall survival rate of patients in the high WDR5 expression group had a significantly lower survival expectation time than those with low WDR5 expression $(\mathrm{P}=0.028$, Fig. 4). In addition, to assess a potential role of WDR5 expression in predicting the postoperative prognosis for patients with $\mathrm{HCC}$, univariate and multivariate analysis was conducted using the Cox proportional hazards model. The results of these analyses are summarized in Table II. The univariate analyses demonstrated that WDR5 expression, histological grade, tumor size and TNM stage were significantly associated with the outcome of HCC. Multivariate analysis indicated that WDR5 expression was an independent prognostic factor, alongside histological grade, tumor size and TNM stage.

\section{Discussion}

The importance of chromatin modification in carcinogenesis has become increasingly evident in recent years (6). Histone modification represents a large proportion of chromatin modifications (6); therefore, the proteins responsible for histone modifications, including methylation, acetylation, phosphorylation, ubiquitylation and ADP ribosylation, have attracted significant attention. Previous studies have demonstrated that the high expression of trimethylated histone $\mathrm{H} 3 \mathrm{~K} 4$ is associated with the prognosis in HCC (21), and that its expression can be regulated by enhancer of zeste homolog 2 (EZH2) (22) or WDR5 (14). EZH2 has been identified as an oncogene in bladder (23), breast (24) and prostate cancer (23). WDR5, the core subunit of the MLL/SET1 complex, is reported as the essential component for complex assembly and methyltransferase activity. WDR5 is also reported to perform a critical role in embryonic stem cell self-renewal and epithelial-mesenchymal transition (7). Furthermore, 

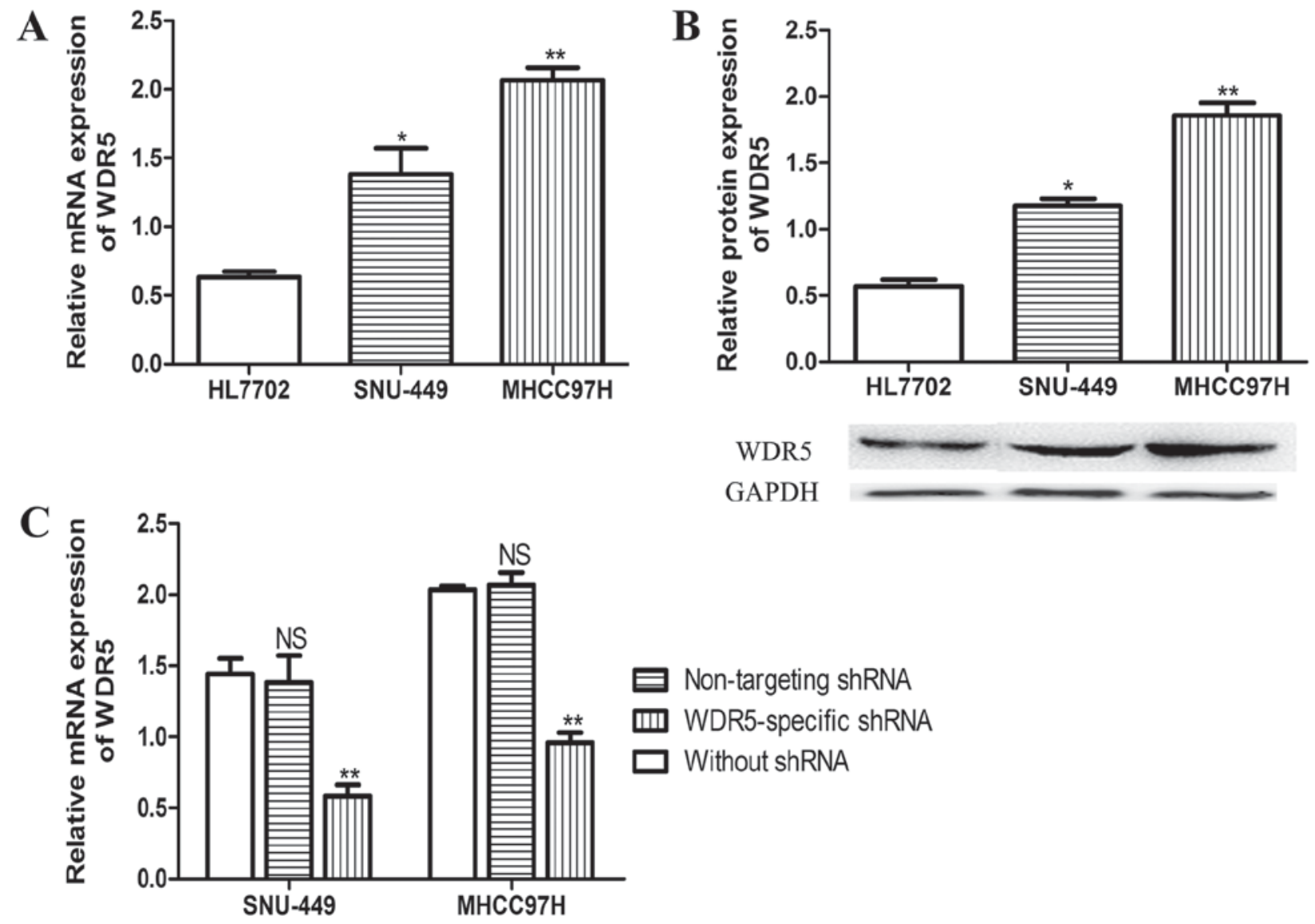

Non-targeting shRNA

而 WDR5-specific shRNA

Without shRNA

Figure 2. WDR5 expression was significantly elevated in HCC cell lines. (A) Reverse transcription-quantitative polymerase chain reaction was used to analyze WDR5 expression in HCC cell lines and a normal liver cell line. (B) Western blotting was used to analyze WDR5 expression in HCC cell lines and a normal liver cell line. ${ }^{*} \mathrm{P}<0.05,{ }^{* *} \mathrm{P}<0.01$ vs. HL7702. (C) shRNA transfection was used to downregulate WDR5 expression in HCC cell lines. ${ }^{* *} \mathrm{P}<0.01$ vs. untransfected group. Statistical analysis was performed with one-way analysis of variance and Tukey's test. WDR5, WD repeat domain 5; HCC, hepatocellular carcinoma; shRNA, short hairpin RNA; NS, not significant.
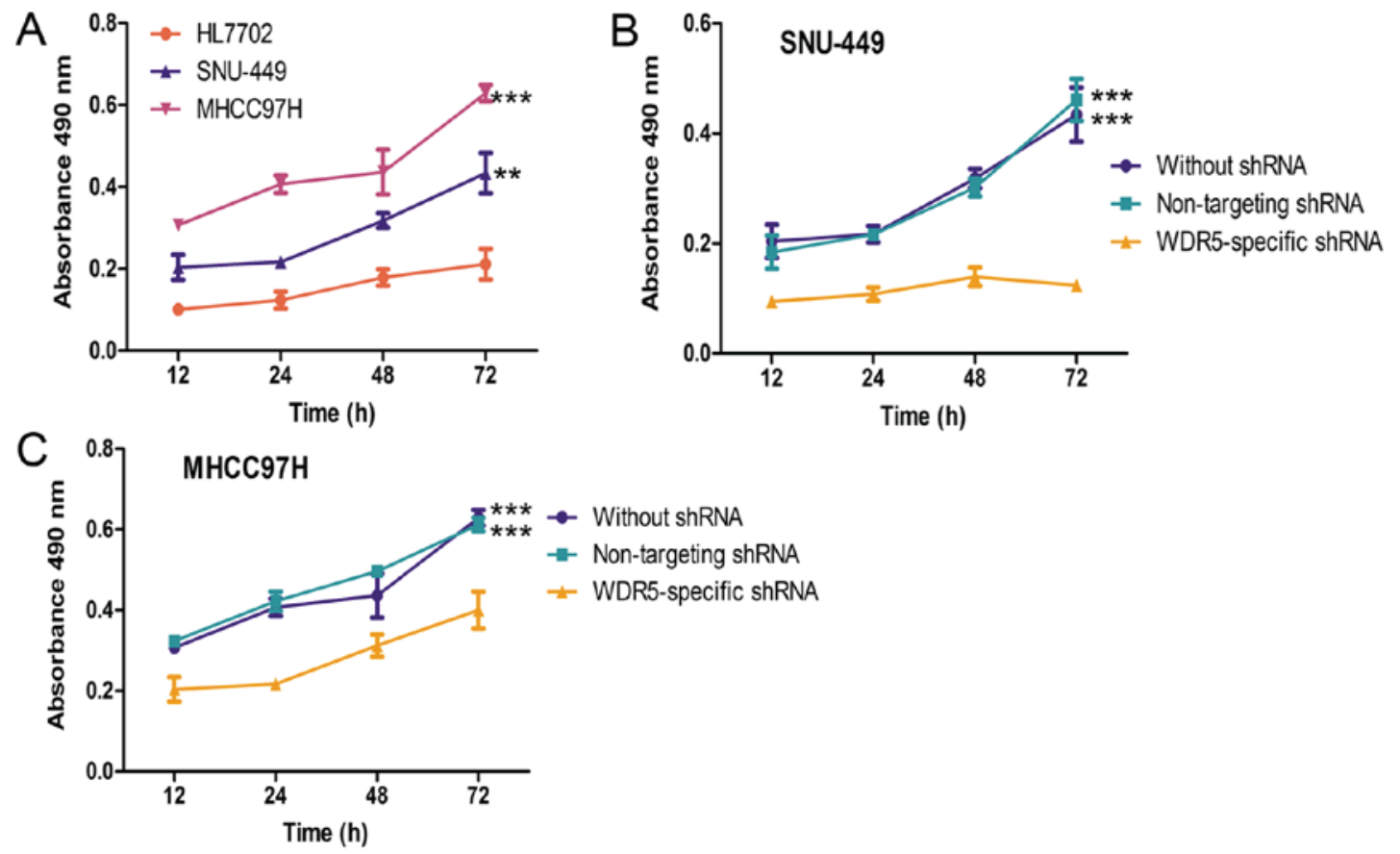

Figure 3. WDR5 expression promotes HCC cell proliferation in vitro. (A) MTT assay to analyze the proliferation rate of HCC cell lines and a normal liver cell line. ${ }^{* *} \mathrm{P}<0.01,{ }^{* * * *} \mathrm{P}<0.001$ vs. HL7702. (B) MTT assay to analyze the proliferation rate of the SNU-449 cell line transfected with non-targeting or WDR5-specific shRNA. (C) MTT assay to analyze the proliferation rate of the MHCC97H cell line transfected with non-targeting or WDR5-specific shRNA. ${ }^{* * * *} \mathrm{P}<0.001$ vs. untransfected group. Statistical analysis was performed with one-way analysis of variance and Tukey's test. WDR5, WD repeat domain 5; HCC, hepatocellular carcinoma; shRNA, short hairpin RNA; NS, not significant. 
Table II. Univariate and multivariate analyses of factors associated with overall survival.

\begin{tabular}{|c|c|c|c|c|c|c|}
\hline \multirow[b]{2}{*}{ Variables } & \multicolumn{2}{|c|}{ Univariate analysis } & \multirow[b]{2}{*}{ P-value } & \multicolumn{2}{|c|}{ Multivariate analysis } & \multirow[b]{2}{*}{ P-value } \\
\hline & HR & $95 \% \mathrm{CI}$ & & HR & $95 \% \mathrm{CI}$ & \\
\hline WDR5 (high vs. low) & 2.151 & $1.141-4.058$ & 0.018 & 2.239 & $1.167-4.296$ & 0.015 \\
\hline Sex (male vs. female) & 1.663 & $0.795-3.479$ & 0.177 & - & - & - \\
\hline Age $(\geq 50$ vs. $<50)$ & 1.755 & $0.850-3.625$ & 0.129 & - & - & - \\
\hline $\operatorname{AFP}(\mathrm{ng} / \mathrm{ml} ; \geq 400$ vs. $<400)$ & 1.948 & $0.977-3.887$ & 0.058 & - & - & - \\
\hline HBsAg (negative vs. positive) & 1.846 & $0.904-3.766$ & 0.092 & - & - & - \\
\hline Liver cirrhosis (no vs. yes) & 1.882 & $0.933-3.795$ & 0.077 & - & - & - \\
\hline Histological grade (well/moderate vs. poor) & 1.921 & $1.014-3.640$ & 0.045 & 1.946 & $1.032-3.671$ & 0.040 \\
\hline Tumor size ( $\geq 5$ vs. $<5)$ & 2.066 & $1.081-3.948$ & 0.028 & 2.081 & $1.094-3.959$ & 0.026 \\
\hline TNM stage (I-II vs. III-IV) & 2.053 & $1.070-3.942$ & 0.031 & 2.007 & $1.046-3.851$ & 0.036 \\
\hline
\end{tabular}

WDR5, WD repeat domain 5; HR, hazard ratio; CI, confidence interval; AFP, $\alpha$-fetoprotein; HBsAg, hepatitis B surface antigen; TNM, tumor-node-metastasis.

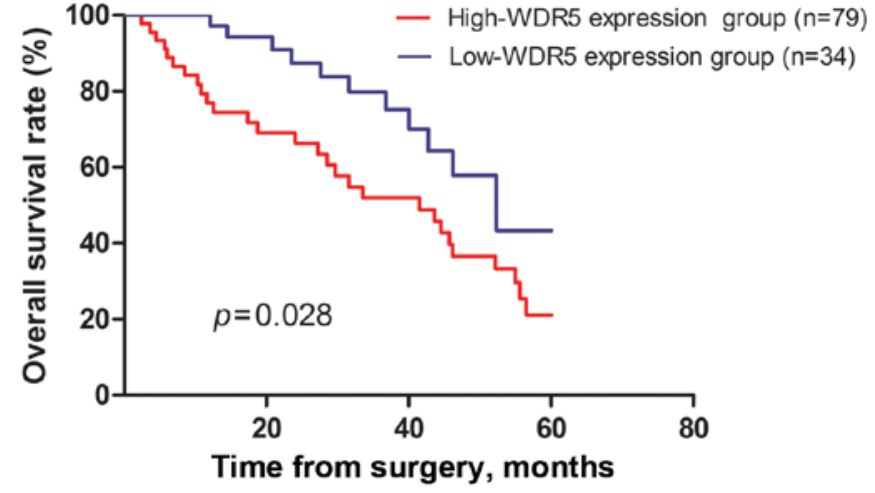

Figure 4. The overall survival rates of the 113 HCC patients were compared in the high-WDR5 and low-WDR5 expression groups. Statistical significance was determined using the log-rank test. HCC, hepatocellular carcinoma; WDR5, WD repeat domain 5.

WDR5 was reported to be overexpressed in prostate (13), bladder (14) and breast cancer (16), and leukemia (15), and was identified as a prognostic indicator in these types of cancer. Additionally, the knockdown of WDR5 expression may result in cell proliferation arrest. However, the WDR5 expression pattern and clinical significance in $\mathrm{HCC}$ are incompletely characterized. Therefore, in the present study, the focus was to investigate the WDR5 expression in HCC tumors, and the association between WDR5 expression and clinicopathological characteristics.

In the present study, the results of RT-qPCR and western blot analysis demonstrated that WDR 5 mRNA and protein expression levels were significantly upregulated in HCC tissue compared with the adjacent normal liver tissues. In order to confirm this, IHC analysis was performed to measure the location and expression of WDR5 in HCC tissue, which demonstrated that WDR5 expression was indeed upregulated in HCC tissue compared with normal adjacent tissue. These findings indicated that WDR5 may serve an important role in HCC.
WDR5 silencing has been reported to reduce the proliferation breast (16) and prostate cancer cells (13). Therefore, to investigate the role of WDR5 expression in the progression of HCC, shRNA-based knockdown was performed to downregulate the expression of WDR5 in HCC cell lines in vitro. These results demonstrated that the HCC cell lines transfected with WDR5-specific shRNA exhibited lower WDR5 expression compared with the wild-type HCC cell lines, which proved the efficacy of WDR5-specific shRNA. An MTT assay indicated that the proliferation rate of HCC cell lines transfected with WDR5-specific shRNA was significantly lower than the wild-type and non-targeting shRNA-transfected HCC cell lines. These findings suggest that WDR5 overexpression promoted cell proliferation.

In addition, the association of WDR5 expression with clinicopathological features and prognosis was investigated. WDR5 expression was associated with tumor size, tumor stage and histological grade based on statistical analysis, indicating the importance of WDR5 in tumor cell growth. The patients were divided into two groups based on the median expression level of WDR5 to determine whether WDR5 expression had prognostic value. The association between WDR5 expression and survival time was assessed by using a Kaplan-Meier curve. Log-rank test analysis demonstrated that the patients with high WDR5 expression had a significantly shorter overall survival time than those with low WDR5 expression. The effects of WDR5 expression and other clinicopathological features on the prognosis of HCC were analyzed using univariate and multivariate analyses. The results indicated that WDR5 expression, histological grade, tumor size and TNM stage were significant predictors for a relatively poor outcome. Conversely, sex, age, serum AFP level, HBsAg, and liver cirrhosis did not significantly affect the outcome of HCC. Notably, multivariate analysis indicated that, in addition to the histological grade, tumor size and TNM stage, WDR5 expression was also an independent predictor for the overall survival of patients with HCC.

In conclusion, WDR5 overexpression is frequently observed in HCC tissues. Furthermore, it was demonstrated WDR5 expression promoted cell proliferation. However, the upstream 
and downstream genes require further study to be identified. In addition, the present study provided clinical evidence that WDR5 tumor expression is positively associated with the poor overall survival of patients with HCC, and may also serve as an independent prognostic factor to determine the outcome of HCC.

\section{Acknowledgements}

Not applicable.

\section{Funding}

No funding was received.

\section{Availability of data and materials}

The datasets used and/or analyzed during the current study are available from the corresponding author on reasonable request.

\section{Authors' contributions}

ZJC and HBL participated in experimental design, interpreted the results and wrote the manuscript; FL, CLM, YHM, XGZ and JDL performed experiments and analyzed the results; FL, CLM and XGZ coordinated the experimental work, interpreted the results and contributed to the critical revision; ZJC and HBL designed the research plan, interpreted the results and wrote the manuscript.

\section{Ethics approval and consent to participate}

The study was approved and monitored by the Research Ethics Committee of the People's Hospital of Rizhao (Rizhao, China). Informed written consent was obtained from all the participating patients.

\section{Consent for publication}

The study participants provided consent for the publication of the present study.

\section{Competing interests}

The authors declare that they have no competing interests.

\section{References}

1. Alejandro F, Josep ML and Jordi B: Hepatocellular carcinoma. Lancet 379: 1245-1255, 2012.

2. Chen WQ, Zheng RS, Baade PD, Zhang SW, Zeng HM, Bray F, Jemal A, Yu XQ and He J: Cancer statistics in China, 2015. CA Cancer J Clin 66: 115-132, 2016.

3. Siegel R, Naishadham D and Jemal A: Cancer statistics, 2013. CA Cancer J Clin 63: 11-30, 2013.

4. Ogunwobi $\mathrm{OO}$ and Liu C: Therapeutic and prognostic importance of epithelial-mesenchymal transition in liver cancers: Insights from experimental models. Crit Rev Oncol Hematol 83: 319-328, 2012.

5. Inokawa $Y$, Nomoto S, Hishida M, Hayashi M, Kanda M, Nishikawa Y, Takeda S, Sugimoto H, Fujii T, Yamada S, Hayashi M, Kanda M, Nishikawa Y, Takeda S, Sugimoto H, Fujii T, Yamada S and Kodera Y et al: Detection of doublecortin domain-containing 2 (DCDC2), a new candidate tumor suppressor gene of hepatocellular carcinoma by triple combination array analysis. J Exp Clin Cancer Res 32: 65, 2013.
6. Bannister AJ and Kouzarides T: Regulation of chromatin by histone modifications. Cell Res 21: 381-395, 2011.

7. Ang YS, Tsai SY, Lee DF, Monk J, Su J, Ratnakumar K, Ding J, Ge Y, Darr H, Chang B, et al: Wdr5 mediates self-renewal and reprogramming via the embryonic stem cell core transcriptional network. Cell 145: 183-197, 2011.

8. Wysocka, J, Swigut T, Milne TA, Dou Y,Zhang X, Burlingame AL, Roeder RG, Brivanlou AH and Allis CD: WDR5 associates with histone $\mathrm{H} 3$ methylated at $\mathrm{K} 4$ and is essential for $\mathrm{H} 3 \mathrm{~K} 4$ methylation and vertebrate development. Cell 121: 859-872, 2005.

9. Trievel RC and Shilatifard A: WDR5, a complexed protein. Nat Struct Mol Biol 16: 678-680, 2009.

10. Dou Y, Milne TA, Ruthenburg AJ, Lee S, Lee JW, Verdine GL, Allis CD and Roeder RG: Regulation of MLL1 H3K4 methyltransferase activity by its core components. Nat Struct Mol Biol 13: 713-719, 2006.

11. Shinsky SA, Hu M, Vought VE, Ng SB, Bamshad MJ, Shendure J and Cosgrove MS: A non-active-site SET domain surface crucial for the interaction of MLL1 and the RbBP5/Ash2L heterodimer within MLL family core complexes. J Mol Biol 426: 2283-2299, 2014.

12. Schuetz A, Allali-Hassani A, Martín F, Loppnau P, Vedadi M, Bochkarev A, Plotnikov AN, Arrowsmith $\mathrm{CH}$ and Min J: Structural basis for molecular recognition and presentation of histone H3 by WDR5. EMBO J 25: 4245-4252, 2006.

13. Kim JY, Banerjee T, Vinckevicius A, Luo Q, Parker JB, Baker MR, Radhakrishnan I, Wei JJ, Barish GD and Chakravarti D: A role for WDR5 in integrating threonine 11 phosphorylation to lysine 4 methylation on histone $\mathrm{H} 3$ during androgen signaling and in prostate cancer. Mol Cell 54: 613-625, 2014.

14. Chen X, Xie WB, Gu P, Cai QQ, Wang B, Xie Y, Dong W, He W, Zhong G, Lin T and Huang J: UpregulatedWDR5 promotes proliferation, self-renewal and chemoresistance in bladder cancer via mediating H3K4 trimethylation. Sci Rep 5: 8293, 2015.

15. Ge Z, Song EJ, Kawasawa YI, Li J, Dovat S and Song C: WDR5 high expression and its effect on tumorigenesis in leukemia. Oncotarget 7: 37740-37754, 2016.

16. Dai X, Guo W, Zhan C, Liu X, Bai Z and Yang Y: WDR5 expression is prognostic of breast cancer outcome. PLoS One 10: e0124964, 2015.

17. Wittekind C, Compton CC, Greene FL and Sobin LH: TNM residual tumor classifcation revisited. Cancer 94: 2511-2516, 2002.

18. Zhou L, Rui JA, Ye DX, Wang SB, Chen SG and Qu Q: Edmondson-steiner grading increases the predictive efficiency of TNM staging for long-term survival of patients with hepatocellular carcinoma after curative resection. World J Surg 32: 1748-1756, 2008

19. Chen Z, Yang P, Li W, He F, Wei J, Zhang T, Zhong J, Chen H and Cao J: Expression of EZH2 is associated with poor outcome in colorectal cancer. Oncol Lett 15: 2953-2961, 2018.

20. Livak KJ and Schmittgen TD: Analysis of relative gene expression data using real-time quantitative PCR and the 2(-Delta Delta C(T)) method. Methods 25: 402-408, 2001.

21. He C, Xu J, Zhang J, Xie D, Ye H, Xiao Z, Cai M, Xu K, Zeng Y, $\mathrm{Li} \mathrm{H}$ and Wang J: High expression of trimethylated histone $\mathrm{H} 3$ lysine 4 is associated with poor prognosis in hepatocellular carcinoma. Hum Pathol 43: 1425-1435, 2012.

22. Margueron R and Reinberg D: The Polycomb complex PRC2 and its mark in life. Nature 469: 343-349, 2011.

23. Weikert S, Christoph F, Kollermann J, Müller M, Schrader M, Miller K and Krause H: Expression levels of the EZH2 polycomb transcriptional repressor correlate with aggressiveness and invasive potential of bladder carcinomas. Int J Mol Med 16: 349-353, 2005.

24. Bachmann IM, Halvorsen OJ, Collett K, Stefansson IM, Straume O, Haukaas SA, Salvesen HB, Otte AP and Akslen LA: EZH2 expression is associated with high proliferation rate and aggressive tumor subgroups in cutaneous melanoma and cancers of the endometrium, prostate, and breast. J Clin Oncol 24: 268-273, 2006.

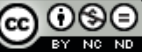

This work is licensed under a Creative Commons Attribution-NonCommercial-NoDerivatives 4.0 International (CC BY-NC-ND 4.0) License. 